\title{
Are solar maximum fan streamers a consequence of twisting sheet structures?
}

\author{
H. Morgan and S. R. Habbal \\ Institute for Astronomy, University of Hawaii, 2680 Woodlawn Drive, Honolulu, HI 96822, USA \\ e-mail: hmorgan@ifa.hawaii.edu
}

Received 18 January 2007 / Accepted 24 February 2007

\begin{abstract}
Context. Fan streamers are often observed at low to mid latitudes in the corona at solar maximum, appearing narrow in latitudinal extent near the Sun, and fanning out with height, adopting an approximately linear, but not necessarily radial, configuration above $\sim 3 R_{\odot}$.

Aims. We offer arguments to support the conjecture that such structures may sometimes consist of high density, non-uniform sheets, viewed edge-on near the Sun, and twisting to a more face-on alignment by $3 R_{\odot}$.

Methods. EUV and white light observations of a fan streamer observed on 2000/12/05 are analyzed. A simple 3D density model is used to recreate the streamer structure.

Results. EIT images show a thin bright sheet at the base of the streamer. The continuation of this structure through the EIT, MLSO MKIV coronameter, and LASCO C2 fields of view, suggests that this sheet is formed mostly of open magnetic field lines. The overall large-scale appearance of the streamer is well simulated by a simple model of a twisting high-density sheet. If the twisting-sheet conjecture is valid, there is a correlation between the distribution of enhanced rays within the streamer viewed in white light, and the distribution of small regions of enhanced brightness seen on the disk in EIT $171 \AA$ at the position of the streamer base.

Conclusions. We suggest that the apparent poleward divergence of equatorial coronal rays, or threads, seen during solar maximum above active regions, may sometimes be a consequence of such a twisting sheet topology.
\end{abstract}

Key words. Sun: corona

\section{Introduction}

The solar maximum corona is extremely complex, and higher resolution observations in the future may reveal increased complexity at smaller scales. Calculating the three-dimensional density structure of such a complex corona is challenging. At the height of solar maximum, bright and filamentary structures are seen at all latitudes, often filling most of the coronal image, with few regions of low brightness, and large reconfigurations in structure can occur from one solar rotation to the next. Large helmet streamers are rare, although many smaller helmet streamers are seen at all latitudes. At low to mid latitudes, fan-shaped structures often appear to emerge from active regions. These structures are highly filamentary, and contain bright rays which, in contrast to helmet streamers, seem to diverge super-radially. Fan streamers have been seen in eclipse observations for many decades (see references in Koutchmy \& Nikoghossian 2002). Observations of equatorial poleward-diverging rays during solar maximum have been described by Eselevich \& Eselevich (2006) as an unexpected phenomenon. Coronal linear threads were studied in detail by Koutchmy \& Nikoghossian (2002) from eclipse and other observations, who show a collection of bright, diverging threads rising from flaring active regions. In this Letter, we provide observational evidence to support the conjecture that fan streamers are a consequence of twisting high density sheet-like structures.

\section{Examples of fan streamers}

The images of Fig. 1 are processed using a recently developed technique, the Normalizing Radial-Graded-Filter (NRGF) (Morgan et al. 2006). Viewing NRGF-processed images is equivalent to viewing a large number of normalized latitudinal profiles simultaenously, thus providing continuity of structure between the fields of view of various instruments. This is the basic means we use to identify fan streamers, and to view their diverging structure, which occurs mostly at heights below the LASCO C2 field of view. The highly complex appearance of the solar maximum corona is apparent from Fig. 1. It is hard to define boundaries between streamers and surrounding structure. Except for a few dark regions of limited latitudinal extent, the whole corona contains filamentary structure of varied brightness. It is difficult to equate the type of bright structure seen here with the more clearly defined helmet streamers of solar minimum.

This work presents an interpretation to explain the appearance of fan streamers, a term we interpret as bright structures which expand from a narrow latitudinal extent at low heights to fill a more significant angular extent at larger heights. Since it is difficult to define boundaries between various structures, fan streamers are not always easy to identify. The white arrows in the images of Fig. 1 point to the bases of fan streamers. They rise from low to mid latitudes, and are associated with active regions at the limb. The brightness across the fan streamers is nonuniform, that is, there are narrow bands of enhanced brightness 

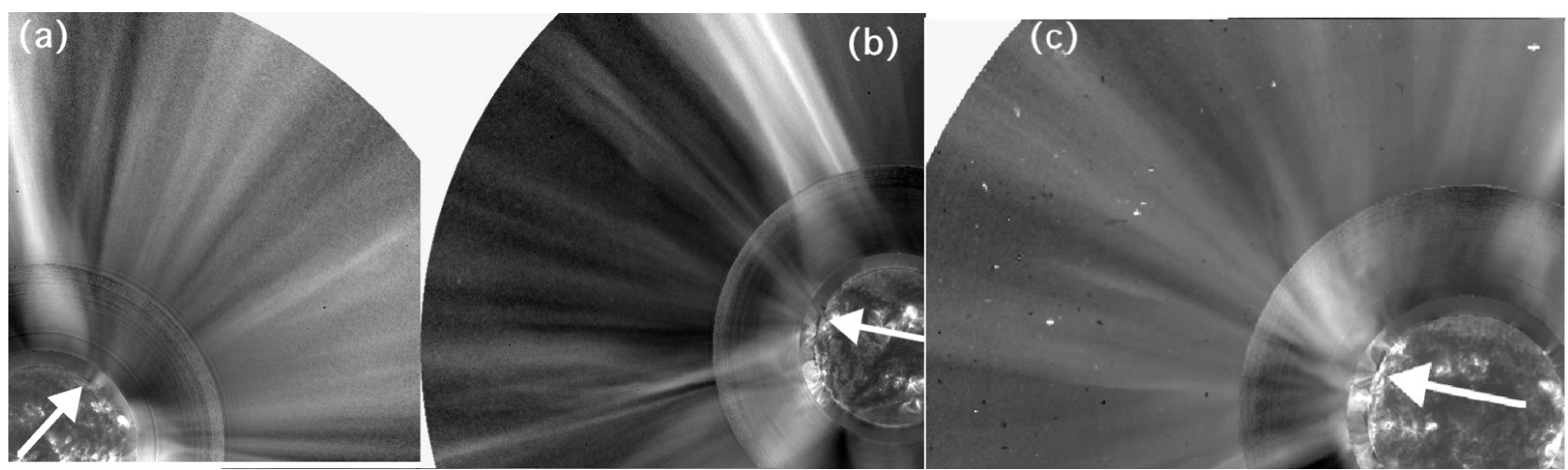

Fig. 1. Fan streamers observed during solar maximum on dates a) 2000/02/13, b) 2000/11/20, and c) 2000/02/24. White arrows point to the bases of the fan streamers. The images are composed of observations by the Solar and Heliospheric Observatory's (SOHO) Extreme Ultraviolet Imaging Telescope (EIT) in the Fe IX/X $171 \AA$ A bandpass at heights $\left(<1.3 R_{\odot}\right)$ (Delaboudiniere et al. 1995), the Mauna Loa Solar Observatory's (MLSO) MKIV coronameter (1.3-2.25 $R_{\odot}$ ) (Fisher et al. 1981) and by the Large Angle and Spectrometric Coronagraph (LASCO) C2 instrument ( $>2.25 R_{\odot}$ ) (Brueckner et al. 1995).

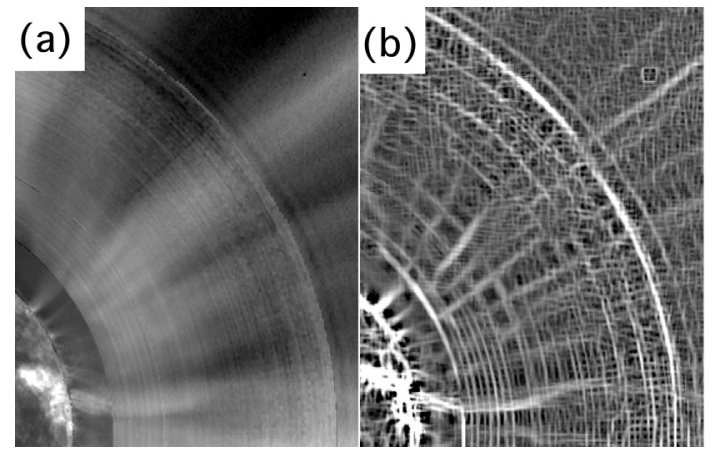

Fig. 2. The north-west corona observed on $2000 / 02 / 24$ by EIT $171 \AA$, MLSO MKIV, and LASCO C2. a) Image processed by the NRGF. b) Same image processed with the madmax filter (Koutchmy et al. 1988, 1992). Rays seen in the NRGF image contain finer threads which are barely discernible, but are revealed by the madmax filter.

which extend continuously through the field of view of MLSO MKIV and LASCO C2. These rays are close to linear (but not necessarily radial) at heights above $\sim 3 R_{\odot}$, but are often bent at lower heights. It is not entirely clear whether these rays are the same features described by Koutchmy \& Nikoghossian (2002) as coronal linear threads, since the eclipse white light images given in that work are processed in a different manner to the images of Fig. 1. Figure $2 \mathrm{a}$ is processed with the NRGF, and Fig. $2 b$ with the NRGF and an edge-enhancement routine. The threads seen in the edge-enhanced image are very similar to the threads described by Koutchmy \& Nikoghossian (2002). Figure 2 shows clearly that the base of these rays, or threads, extends down to the solar surface, as seen in the EIT field of view. That fan streamers are not necessarily associated with closed field regions, and are a common feature during solar maximum, is evident from Figs. 1 and 2 .

\section{Fan streamers as twisting sheets}

The top image of Fig. 3 shows an observation of a fan streamer made during 2000/12/05. It is apparent at the limb for $\sim 3$ days, and appears half a solar rotation later at the west limb, altered, but still fan-shaped. The streamer shows a super-radial divergence at heights below $\sim 3 R_{\odot}$. This expansion is symmetrical around the central axis of the streamer. We suggest that the general structure of this streamer is a high-density twisting sheet,
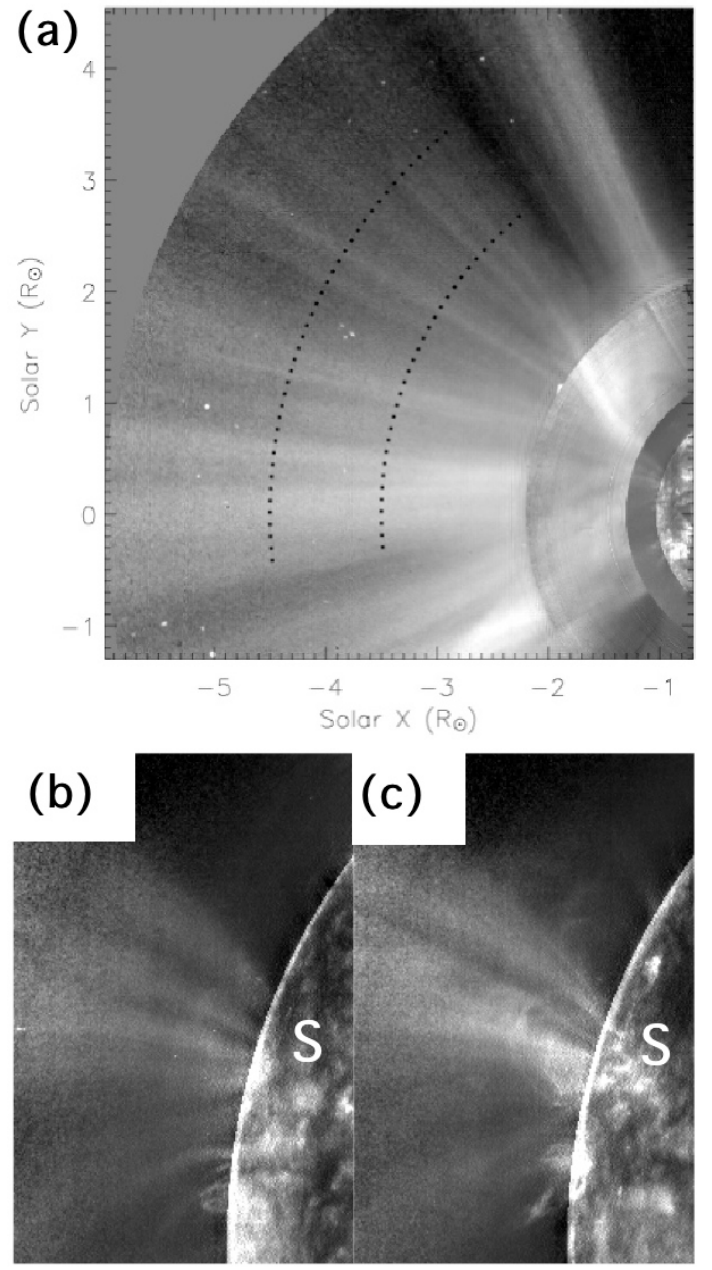

Fig. 3. a) Equatorial fan streamer observed during 2000/12/05. The image is a composite of EIT $171 \AA$, MKIV and LASCO C2 $p B$ observations. The two dotted lines are at heights of 3.5 and $4.5 R_{\odot}$, and are of relevance to Fig. 4. b), c) Detail of the equatorial streamer at low heights, observed by EIT in $171 \AA$ at a) 19:00 on 2000/12/05, and b) 19:00 on 2000/12/06.

with threads of higher density embedded in the sheet forming the rays. The sheet at low heights in the EIT image is seen close to edge-on, and appears bright and narrow. Between the Sun and 

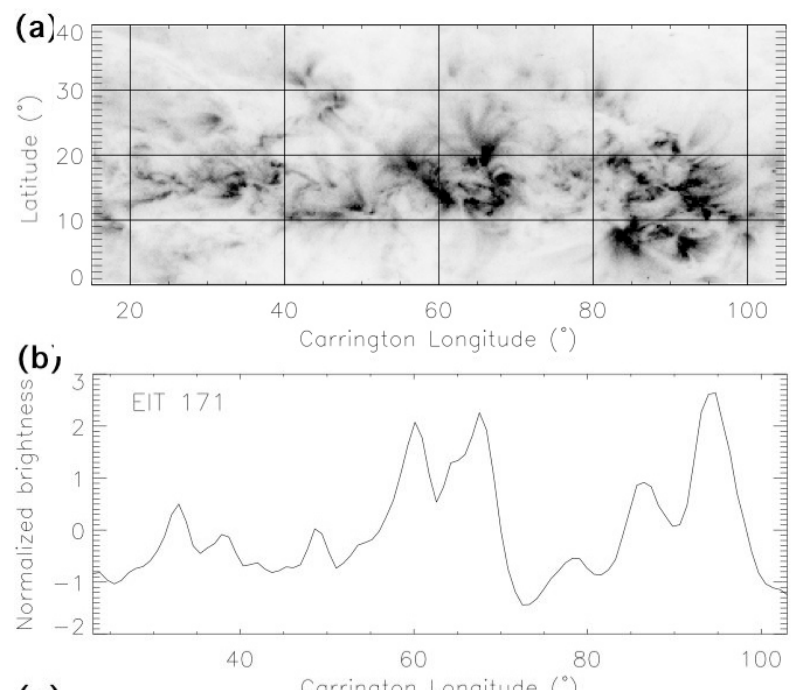

(c)

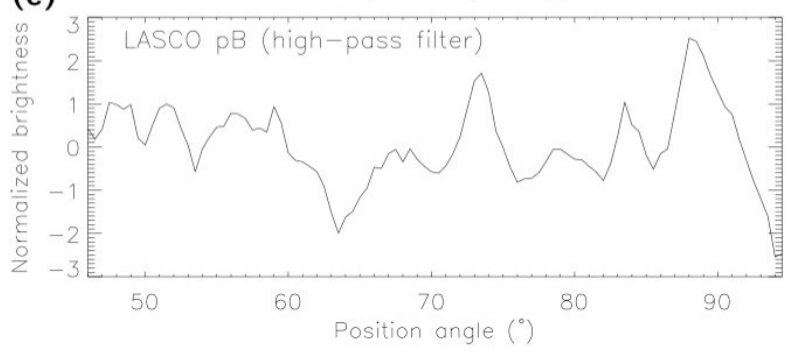

Fig. 4. a) EIT $171 \AA$ map of the source region of the fan streamer of Fig. 3, observed during 2000/12/16. The brightness is reversed (black regions have the highest intensity). b) Brightness of the EIT map integrated over latitudes $12^{\circ}-18^{\circ}$, given in arbitrary units. c) Finescale structure (or enhanced rays) observed in $p B$ across the equatorial streamer observed by LASCO C2 during 2000/12/05. To increase the signal to noise, many latitudinal profiles of $p B$ between heights of 3.5$4.5 R_{\odot}$ are normalized to a mean of zero and a standard deviation of one and summed (see Fig. 3a). The resulting latitudinal profile is fitted to a 2-degree polynomial. This smooth fit is subtracted from the original to reveal the smaller scale variations across the streamer.

$\sim 3 R_{\odot}$, the sheet twists to a more face-on alignment. This is a simple topology which can explain the apparent non-linear divergence or expansion of the fan streamer.

More details of the fan streamer as seen by EIT $171 \AA$ are shown in Figs. 3b, c. A collection of rays rise from an active region complex labeled $\mathrm{S}$, which we call the source region. In Fig. 3b, the source region is mostly hidden behind the limb, although we can see the rays at heights above the limb. Figure $3 c$ shows that part of the source region has become visible on the disk. Also apparent in Fig. 3c is a small system of closed loops, existing alongside the open rays above the source region. By 2000/12/16, the source region is fully revealed in EIT, centered at the meridian. This region is shown in the longitudelatitude map of Fig. 4a. A chain of active regions extends from Carrington longitude $\sim 30^{\circ}-100^{\circ}$, limited in latitude between $\sim 10^{\circ}-20^{\circ}$.

Figure $4 \mathrm{~b}$ shows the profile of intensity across the EIT source region map, integrated across pixels between latitudes of $12^{\circ}-$ $18^{\circ}$. This plot serves to show the position of the most active spots across the source region, plotted against Carrington longitude. Figure $4 \mathrm{c}$ shows the position of the enhanced rays across the fan streamer as seen by LASCO C2, plotted against position angle. Given that some unknown proportion of the active region brightness seen on the disk in EIT arises from closed field structures, there is still reasonable correlation between the EIT and LASCO profiles to the right of these plots (between longitudes $55^{\circ}-100^{\circ}$ in Fig. $4 \mathrm{~b}$, or position angles $65^{\circ}-95^{\circ}$ in Fig. $4 \mathrm{c}$ ), and this is supportive of the twisting-sheet interpretation of the streamer structure. The correlation between the EIT and LASCO profiles across this section of the streamer is close to $70 \%$. The sheet emerging from the westward side of the source region twists southwards, forming the edge of the fan streamer near the equator. This is the part which shows the strongest correlation. The eastward part of the sheet twists to the north, forming the north-east edge of the fan streamer.

To lend support to this interpretation we construct a model where we place a twisted, high-density sheet, of thickness $4^{\circ}$, within a uniform $3 \mathrm{D}$ grid. The model is very similar to that described in detail by Morgan et al. (2007), and is conceptually similar to other works aimed at interpreting structures within the corona (Vibert et al. 1997; Thernisien \& Howard 2006, for example). The base of the sheet at the solar surface extends from longitudes $30^{\circ}$ to $100^{\circ}$ at a constant latitude of $15^{\circ}$, corresponding to the observed source region shown in Fig. $4 \mathrm{a}$. With increasing height, the sheet is twisted, until a final radial configuration is reached at $3 R_{\odot}$, with an inclination of $60^{\circ}$ to east-west. Figure 5 shows the surface (red) and final (yellow) configuration of the model streamer overlaid on a Wilcox Solar Observatory (WSO) photospheric magnetic field map. The density outside of the streamer is zero. Within the streamer, the density profile across the sheet (in arbitrary units) is fixed by the profile of observed LASCO $p B$ variation across the active region (as the normalized profile shown in the middle plot of Fig. 4c, but increased to a minimum value of 1 to avoid negative values). We obtain $p B$ images (again in arbitrary units) from the density model by integrating along lines of sight within the uniform grid (van de Hulst 1950; Quémerais \& Lamy 2002), with geometrical alignments (or coordinates within the grid) equivalent to the observations made on 2000/12/05. The radial gradient of brightness within the image is then removed using the NRGF. It is this step which allows a qualitative comparison of modeled and observed structures, which are also processed with the NRGF. The NRGF, which flattens a coronal image into a set of normalized latitudinal profiles, allows a non-rigorous study of structure without the calculation of absolute density.

The comparison between the modeled and observed images is shown in Fig. 6. The complicated diverging shape of the observed fan streamer is well replicated by the simple twisting sheet structure. Although the overall shape and the distribution of rays within the model streamer are not matched exactly with the data, this could be easily improved by fine-tuning the model parameters, such as the position of the rotation axis of the twisting sheet, the relative densities of the rays within the streamer, the rate of twist at lower heights, and the shape of the modeled sheet. For the sake of simplicity, we have assumed that the sheet follows a straight line in longitude-latitude space at a given height. However, from the discrepancies between the model and observation, the sheet is likely to have some curvature. Given the deliberate simplicity of the model, the agreement is good, showing that this fan streamer is very likely to be a large twisting sheet structure.

\section{Discussion}

The existence of high-density thin sheets in the corona is well known (Sturrock \& Smith 1968; Thernisien \& Howard 2006, for example). That they emerge from active regions is also not 


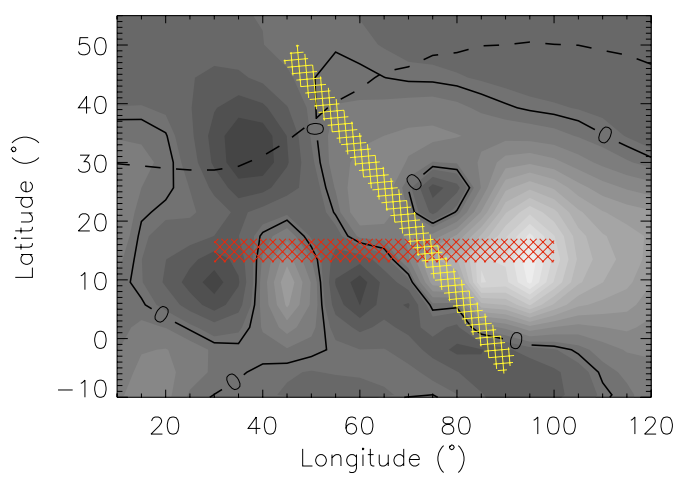

Fig. 5. Modeled configuration of the equatorial streamer, overlaid on a synoptic map of the photospheric magnetic field measured by WSO. The streamer base at the Sun is shown as a red region, and the final radial configuration shown as the yellow region. Brighter regions in the photospheric field map show a stronger positive field. Neutral lines are shown as solid bold contours. The dashed bold contour shows the neutral line at a height of $2.5 R_{\odot}$, as calculated by WSO using a PFSS extrapolation of the photospheric field.

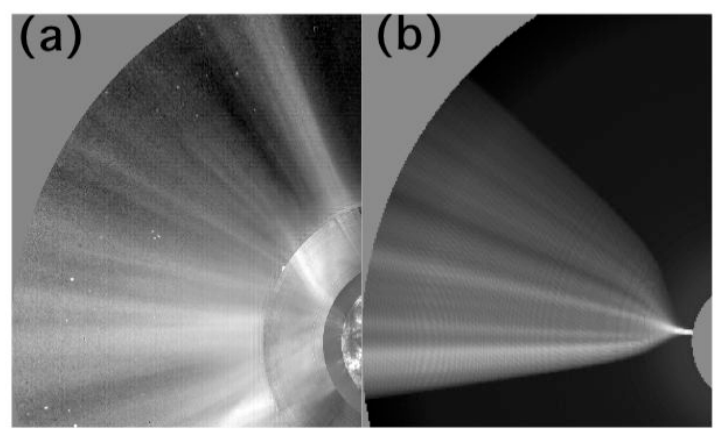

Fig. 6. a) Observed and b) modeled images of the equatorial streamer for date 2000/12/05. The central meridian is at a longitude of $203^{\circ}$, with the east limb at a longitude of $113^{\circ}$. The source region of the equatorial streamer is at longitude $\sim 30^{\circ}-100^{\circ}$, hidden just behind the east limb.

surprising. These structures are usually associated with, or contain, magnetic neutral sheets, which are expected to form above the cusps of large closed magnetic field regions, where streams of open field emerging from surrounding regions of opposite polarity converge into a stalk (Newkirk 1967, for example). The present work provides evidence that a high-density sheet need not always form above an arcade of closed loops, but may form very low in the corona, or even below. It is unclear wether this high-density sheet traces a boundary between regions of opposite magnetic polarity. The position of the neutral line in the corona, calculated by a potential field source surface (PFSS) extrapolation of the observed photospheric field (Altschuler \& Newkirk 1969; Wang \& Sheeley 1992) is shown in Fig. 5 as a dashed bold line. The approximate configuration of the fan streamer sheet in the corona is given by the yellow band, which bears no relation to the PFSS neutral line. This is not entirely surprising, since PFSS calculations cannot account for rapid changes in the photosphere during solar maximum (Wang et al. 2000). Liewer et al. (2001) considered that during times outside solar minimum, more filamentary structure, not predicted by the uniform heliospheric current sheet of PFSS models, is produced by newly opened field lines created by reconnection processes in active regions.
One possible mechanism for the creation of large-scale twisting sheets is the likely rigid rotation of the corona above the differential rotation of the photosphere (Howard 1984; Lewis et al. 1999), which would force the deformation, or twisting, of structure. The differential rotation would demand a time-dependent deformity, with twists increasing or decreasing with time, depending on the particular geometry (alignment and latitude) of the structure.

Fan streamers may be more common at low latitudes because chains of active regions in narrow latitudinal bands, but extended in longitude, are more common in these regions. There are also geometrical line of sight considerations. To achieve a fluted shape with diverging rays, a twisted sheet must appear narrow, or must be seen edge-on, near the Sun. A high-density sheet rising from a constant latitude from the Sun is a flatter surface when near to the equator, and would appear bright and narrow when seen from Earth. At increasing latitudes, such a surface becomes curved like a section of a cone, and would not be apparent as a thin structure in observations.

\section{Summary}

Fan streamers are a common feature of the solar maximum corona, and they extend all the way to the solar surface. We interpret the structure of the fan streamer of 2000/12/05 as a sheet of high density material, emerging directly from a extended chain of active regions at low latitude. The twisting of the high-density sheet with height recreates the diverging fan shape of the streamer at heights below $3 R_{\odot}$. If we accept this interpretation of structure, the distribution of enhanced brightness rays within the sheet correlate with the position of spots of enhanced brightness along the source region on the disk.

Acknowledgements. The $p B$ data used in this work are courtesy of the MLSO and the LASCO/SOHO consortium. The MLSO coronagraph is operated by HAO, a division of the NCAR, which is sponsored by the NSF. SOHO is a mission of international cooperation between ESA and NASA. The WSO data were obtained from the Stanford University website courtesy of J. T. Hoeksema. WSO is supported by NASA, the NSF and ONR.

\section{References}

Altschuler, M. D., \& Newkirk, G., Jr. 1969, Sol. Phys., 9, 131

Brueckner, G. E., Howard, R. A., Koomen, M. J., et al. 1995, Sol. Phys., 162, 357

Delaboudiniere, J.-P., et al. 1995, Sol. Phys., 162, 291

Eselevich, M. V., \& Eselevich, V. G. 2006, Sol. Phys., 235, 331

Fisher, R. R., Lee, R. H., MacQueen, R. M., \& Poland, A. I. 1981, Appl. Opt. 20, 1094

Howard, R. 1984, ARA\&A, 22, 131

Koutchmy, O., Koutchmy, S., Nitschelm, C., Sykora, J., \& Smartt, R. N. 1988, Solar and Stellar Coronal Structure and Dynamics, 256

Koutchmy, S., \& Nikoghossian, A. G. 2002, A\&A, 395, 983

Koutchmy, S., Altrock, R. C., Darvann, T. A., et al. 1992, A\&AS, 96, 169

Lewis, D. J., Simnett, G. M., Brueckner, G. E., et al. 1999, Sol. Phys., 184, 297

Liewer, P. C., Hall, J. R., De Jong, M., et al. 2001, J. Geophys. Res., 106, 15903

Morgan, H., \& Habbal, S. R. 2007, A\&A, 464, 357

Morgan, H., Habbal, S. R., \& Woo, R. 2006, Sol. Phys., 236, 263

Newkirk, G. J. 1967, ARA\&A, 5, 213

Quémerais, E., \& Lamy, P. 2002, A\&A, 393, 295

Sturrock, P. A., \& Smith, S. M. 1968, Sol. Phys., 5, 87

Thernisien, A. F., \& Howard, R. A. 2006, ApJ, 642, 523

van de Hulst, H. C. 1950, Bull. Astron. Inst. Netherl., 11, 135

Vibert, D., Lamy, P., \& Liebaria, A. 1997, ESA SP-404: Fifth SOHO Workshop:

The Corona and Solar Wind Near Minimum Activity, 713

Wang, Y.-M., \& Sheeley, N. R., Jr. 1992, ApJ, 392, 310

Wang, Y.-M., Sheeley, N. R., Jr., \& Rich, N. B. 2000, Geophys. Res. Lett., 27, 149 